\title{
Development of the Skin of the Surface of the Tunnel Part of the Ship's Hull
}

\author{
Irina Shorkina ${ }^{1,2}$ and Sergey Novikov ${ }^{1,3}$ \\ ${ }^{1}$ Volga State University of Water Transport, Nesterova st., 5, Nizhny Novgorod, 603951, Russia \\ ${ }^{2}$ Nizhegorodsky State Architectural and Civil Engineering University, Ilyinskaya st., 65, Nizhny Novgorod, \\ 603950, Russia \\ ${ }^{3}$ Samara State Transport University, Svobody st., 28, Samara, 443066, Russia
}

\begin{abstract}
In modern shipbuilding, extremely high requirements are imposed on the speed of the vessel. The material costs for ensuring the seaworthiness of modern transport vessels account for about half of the constant operating costs. Experimental materials confirm that the geometry of the surface of the tunnel part of the vessel's hull has a significant effect on its propulsive coefficient. Improving the shape of the tunnel part allows you to change the hydrodynamic pressure and reduce the negative effect of the flow formed when the vessel moves forward. At the same time, the complex geometric shape of this part of the hull significantly complicates the preparation of maps of its cutting and, as a result, its production, since it is a set of complex surfaces of double curvature. This article describes a practical approach to solving the problem of constructing a sheet cutting map. For modeling a three-dimensional model and controlling the shape of the surface, the capabilities of the Rhinoceros 6 system are presented. The results are presented as the deviation in the area of the deployable and non-deployable surfaces.
\end{abstract}

\section{Keywords}

Surfaces of double curvature, surface unfolding, surface cutting maps, surface quality, curvature visualization, the shape of the aft end of the vessel

\section{Introduction}

In recent decades, the shipbuilding industry has been intensively searching for alternative architectural forms of ship hulls. One of the priority studies in this direction is the design of tunnel-type vessels $[2,3]$. The main research is aimed at finding ways to improve the main geometric characteristics of the hull with single-hull and multi-hull architecture of the vessel, optimizing the geometry of the contours for specific operating conditions $[4,5]$. However, in the vast majority of cases, one of the main problems in this case is the construction of unrolling sheets of the shell of the vessel with a complex shape of the stern contours and the subsequent construction of material cutting maps.

The purpose of this work is the practical construction of the unrolling of the sheets of the shell of the tunnel part of the ship's hull. The geometric problem of constructing surface scans is the basic one when forming material cutting maps. Numerous studies have been devoted to solving the problem of unrolling surfaces of single and double curvature onto a flat region with minimal deviations in surface area, see, for example, $[7,8]$. 


\section{Description of the approach to solving the problem}

In accordance with the point of view presented in [1], the problem of surface unrolling is divided into two independent formulations, namely: the construction of an unstrained scan of ruled surfaces and the construction of a mapping of non-deployable surfaces of double curvature to a flat area, which is associated with some deformation of surface elements. A more detailed study of the problem shows that from a geometric point of view, both formulations do not differ from each other and can be considered as an isometric mapping of a surface to a flat area. As is known, the isometric mapping of a surface is also equiareal and conformal due to the conservation of both the areas of the elementary parts of the surface and the angles between an arbitrary pair of curves on the surface. The only difference is that linear surfaces are displayed without distortion of linear elements, and surfaces of double curvature can be mapped to a flat area only approximately.

In this work, the problem of forming the unrolling sheets of the tunnel shell of the hull of the passenger ship - the floating restaurant "Zhemchuzhina" was solved. The passenger vessel was built by reconstructing and re-equipping the hull of a self-propelled ferry with a lifting capacity of 60 tons. The self-propelled ferry "Gidrolog" had a rounded stern and one water jet propulsion, intended for carrying out various types of work on rivers. The hull of the passenger vessel was designed with a transom stern and two tunnel formations in the stern to accommodate the propeller-steering complex.

As initial information, the customer provided a theoretical drawing (in DXF format) of the tunnel of the updated passenger ship hull (fig. 1). To form the surface, the initial data can be represented as a certain cloud of points in the coordinate system "Theoretical frames - Theoretical Waterlines", or can be represented as lines of a theoretical drawing (wire model), or both together.
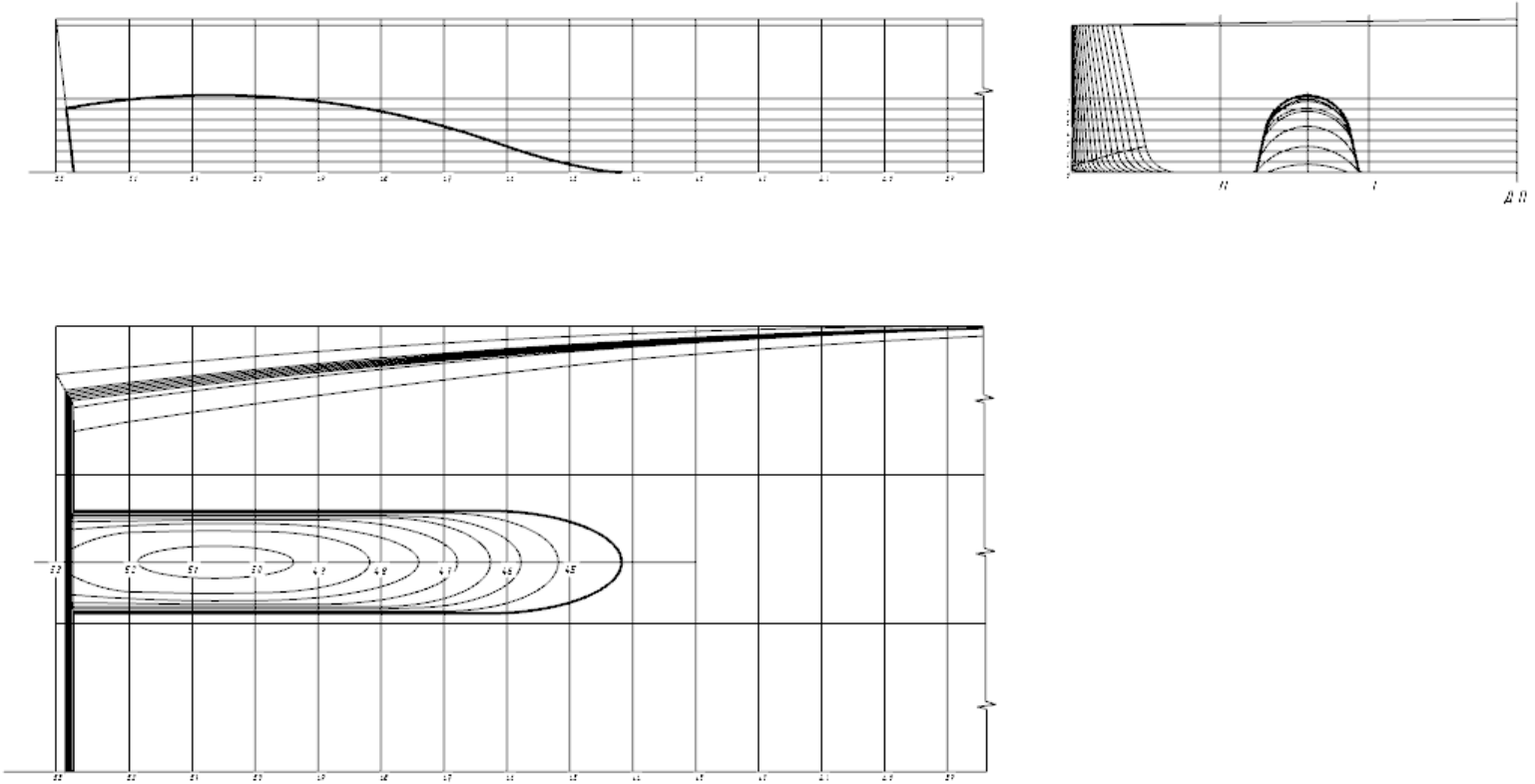

Figure 1: Theoretical drawing of the tunnel

Modeling of the tunnel surface and the formation of skin scans was performed using the capabilities of the Rhinoceros 6 graphics package [9].

The Rhinoceros 6 graphics package has a set of tools that allows you to create, edit, analyze, visualize and transform NURBS curves, surfaces, solids, point clouds and polygon networks with high accuracy. The complexity of the surface or the size of the object do not impose boundary conditions for the formation of smooth models, deprivation can arise only from the computer technology used.

To solve this problem, an algorithm for constructing a cutting map was formulated:

\section{Formation of a three-dimensional model of the tunnel surface.}

The model was created based on a network of curves using the Surface Curve Network command. The set of curves in the first direction was set by the contours of the tunnel frames, and in the second direction - by the contour of the zero waterline and the zero buttock-line of the tunnel. Between the 
surface of the tunnel and the bottom there is a zygomatic rounding with a radius of $50 \mathrm{~mm}$, which was created by the Fillet Surface team. The resulting model is shown in figure 2.
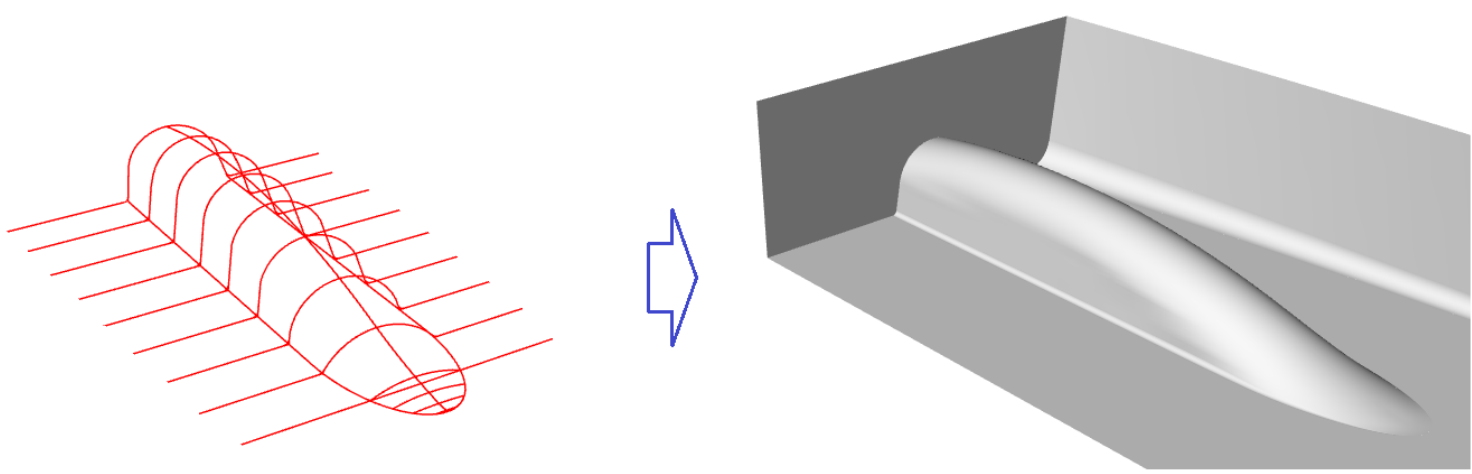

Figure 2: Model of the tunnel surface based on a network of curves using the Surface Curve Network command

\section{Analysis of the curvature of the surface.}

To form the cutting maps, the curvature of the tunnel surface was analyzed. Currently, several methods of quality control are used for ship surfaces. Quality control methods make it possible to see the change in the geometry of the surface sections, allow you to identify the kinks of the curve and monitor the transition areas of curved contours to flat ones. The methods allow us to find the best surface that is close to the original data with minimal deviation, so that the surface satisfies the conditions of smoothness and monotony. The use of control methods gives a real representation of the form, which will meet the quality criterion as much as possible.

The Rhinoceros 6 graphics package has tools that allow you to control the quality of the shape of the simulated surface by visualizing the Gaussian curvature of the surface and the curvature of curves and sections:

- visualization of Gaussian curvature. The method clearly reflects the quality of the surface due to the color transition. A sharp change in color contrast indicates problems with the smoothness of the surface in the area under consideration;

- visualization of the curvature of curves, sections and surfaces. Curvature visualization allows you to check the most difficult sections of curves. If the curvature plot is continuous and changes smoothly, then the characteristics are desirable for the curve and the curve is smoothed. Jumps in the curvature plot indicate kinks.

The Gaussian curvature toolkit shows the colors on the surface, based on the average curvature at each point (figure 3). The color here is not as important as the shape that creates the color. When

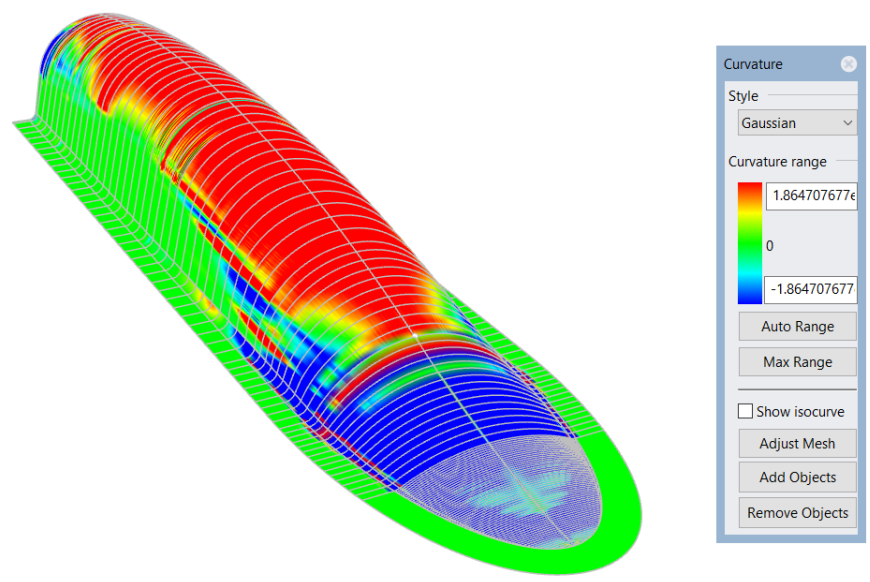

Figure 3: Visualization of the Gaussian curvature of the surface. The Gaussian curvature tool 
the color progressions are gradual, the surface shows an acceptable degree of smoothing. Analyzing the surface, areas were identified that require additional testing for smoothness.

To clarify the display of changes in the curvature of curves on some parts of the surface, graphs of the curvature of sections were constructed. The curvature plot showed the characteristics required for the curve. The plot changed smoothly, there were no jumps in the curvature plot, therefore, the curve satisfies the conditions. Figure 4 shows a plot of one cross-sectional curve, where the smoothness of the curve is clearly visible.

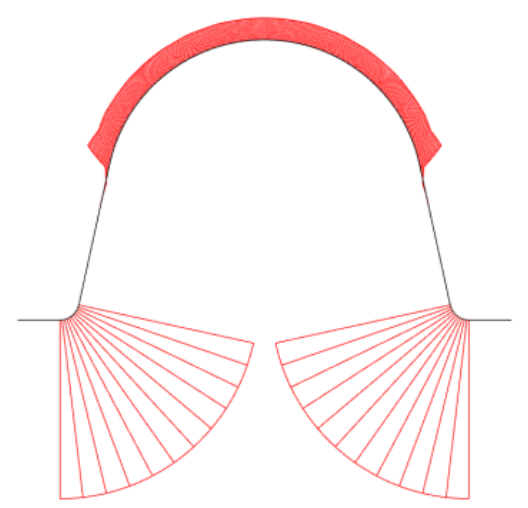

Figure 4: Analysis of the curvature of the cross-section line

\section{Formation of unfolding sheets of tunnel skin.}

To form the unfolding of the skin sheets, the simulated surface was divided into nine parts (fig. 5) with planes parallel to the plane of the midship frame and offset $100 \mathrm{~mm}$ aft from the elements of the transverse tunnel set.

single curvature shell fragments

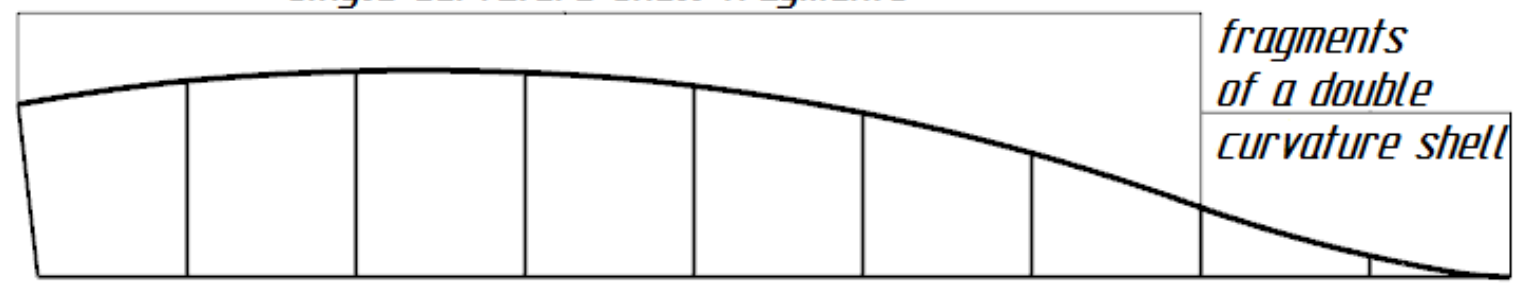

Figure 5: Splitting the tunnel surface into parts

Seven parts of the tunnel surface were defined as ruled surfaces and the construction of the skin sheets was reduced to the construction of an unstressed sweep of single curvature. The two parts of the tunnel surface are surfaces of double curvature. These fragments of the skin were further divided into parts in order to minimize the deviation of the sweep area from the area of the original surface.

One of the factors affecting the transport efficiency of the vessel is the approach to constructing the surface scans of single and double curvature on a flat area. The solution of questions concerning geometric methods for constructing optimal surface scans is proposed in $[6,7,11]$. The construction of an unstressed scan of ruled surfaces and the construction of a mapping of non-deployable surfaces of double curvature to a flat area is associated with some deformation of the surface elements. From a geometric point of view, the construction of both scans is considered as an isometric mapping of the surface to a flat area. As described in [10], the isometric mapping of a surface is equiareal and conformal due to the conservation of both the areas of the elementary parts of the surface and the angles between an arbitrary pair of curves on the surface. According to this statement, when performing a sweep on a plane, it is necessary to meet the requirements for preserving the lengths of the sides and minimize the difference in the areas of the original surface with the sweep area.

The scan of the received fragments of the surface of single curvature was performed by the Unroll Developable Surface team. In order to unfold a surface with this tool, the surfaces must be linear in one 
direction. The Gaussian curvature of each fragment is almost zero at each point of the surface. This tool is best suited for building an unstressed scan.

Construction of scans of fragments of the double curvature skin is not an easy task. To solve this problem, many schemes have been developed in Rhinoceros 6 that claim to transfer complex computational geometry to the physical world. To form the cut, two forward fragments were conditionally unrolled using the Smash Double-Curved Surface command. The most forward fragment of the skin was further divided into two parts in order to minimize the deviation of the sweep area from the area of the original surface (figure 6).

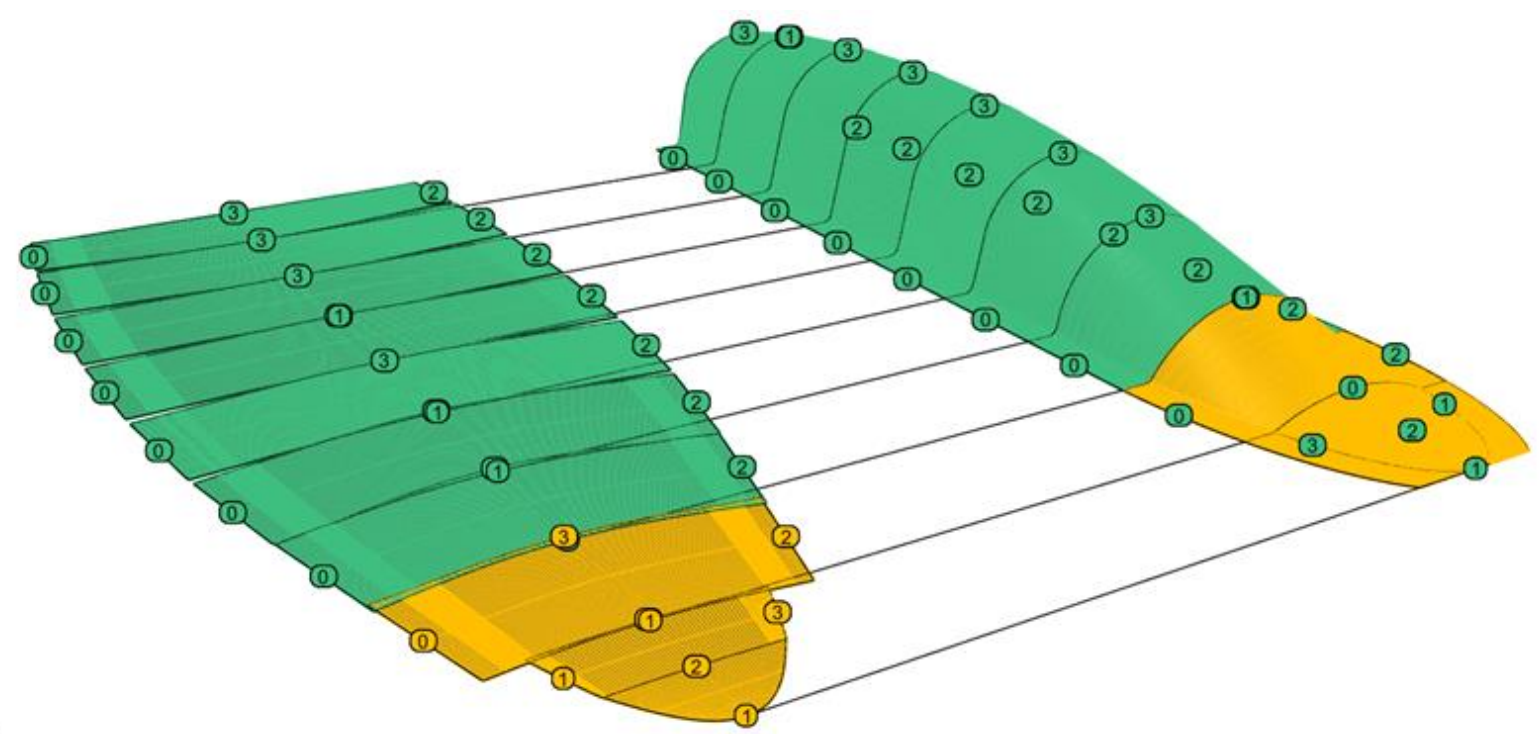

Figure 6: Scanning the tunnel skin onto a flat area

\section{Results}

As a result of the performed operations, a 1:1 scale cutting map of the skin sheets of the tunnel part in DXF format was formed (figure 7). The file was transferred to the customer for cutting steel sheets on a CNC machine.

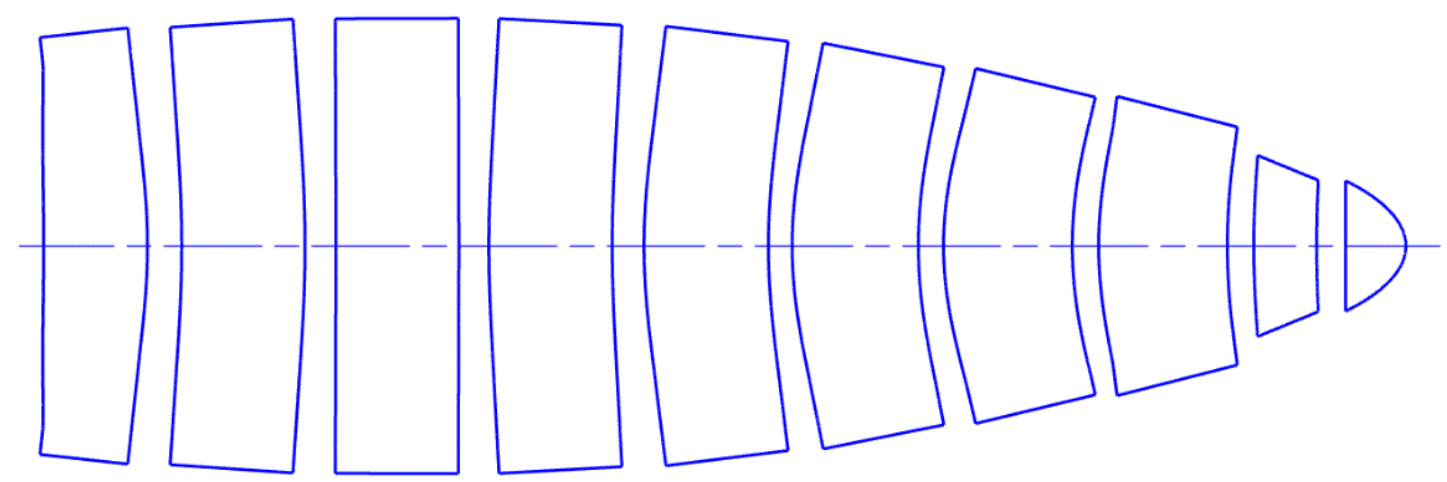

Figure 7: The tunnel part cutting map

The analysis of the results of the construction of scans, both of linear deployable surfaces and of surfaces of double curvature, showed that the maximum deviation in area was less than $1 \%$ (Table 1 ). It should be noted that the surface was scanned without taking into account the thickness of the steel shell, which is $5 \mathrm{~mm}$. 
Table 1

Results of building scans

\begin{tabular}{ccccc}
\hline & $\begin{array}{c}\text { Surface area, } \\
\mathrm{mm}^{2}\end{array}$ & $\begin{array}{c}\text { Scan area, } \\
\mathrm{mm}^{2}\end{array}$ & $\begin{array}{c}\text { Area difference, } \\
\mathrm{mm}^{2}\end{array}$ & $\begin{array}{c}\text { Area difference, } \\
\%\end{array}$ \\
\hline $\begin{array}{c}\text { Deployable } \\
\text { surface }\end{array}$ & 1252589,880 & 1248312,400 & 4277,480 & 0,341490864 \\
$\begin{array}{c}\text { Non-deployable } \\
\text { surface }\end{array}$ & 809004,412 & 806480,075 & 2524,337 & 0,312030066 \\
\hline
\end{tabular}

The adopted design solutions turned out to be mostly correct and fully meet the customer's requirements. The formation of the shell and its installation to the elements of the ship's hull set did not cause difficulties for the shipyard workers.

\section{References}

[1] A.V. Pogorelov, Differential Geometry, 6th ed., Moscow, Nauka, 1974.

[2] A. Pylaev, Tunnel type ship, 2019. URL: http://unionexpert.su/korabl-tunnelnogo-tipa/

[3] Yu. Makarov, A. Mozgovoy, In Search of New Solutions, 2017. URL: https://oborona.ru/includes/periodics/navy/2017/0725/104321914/detail.shtml

[4] A.B. Petrov, Efficiency of oversized propellers on ships with a tunnel stern bypass, Master' thesis, Leningrad, USSR, 1985.

[5] V.P. Lang, V.F. Belonenko, K.A. Smirnov, Displacement Semi-Trimaran Corps, 2014. Patent No. RU2014100993A, Filed January 15, 2014, Issued July 20. URL: https://patents.google.com/patent/RU2014100993A/ru

[6] E.V. Popov, Construction of sweeps of surfaces of single and double curvature, Descriptive geometry, engineering and computer graphics: International interuniversity collection, works of the departments of graphic disciplines, N. Novgorod: NGASU, 2000, Issue 5, pp. 272-276.

[7] V.N. Shalimov, K. Shalimova, Algorithm for constructing cutting maps for awning fabric structures, volume 27 Collection of scientific papers Sworld, No. 1, Ivanovo: Scientific world, 2010, pp. 37-40.

[8] A. Kuznetsov, Yu. Platonov, A. Ryabokon, Specialized CAD tools for shipbuilding by "CSoft Bureau ESG" based on Autodesk products, CAD \& Graphics magazine, volume 6, 2012. URL: https://sapr.ru/article/23075

[9] New features in Rhino 6, 2021. URL: https://www.rhino-3d.ru/rhinoceros/new-in-rhino6/features/

[10] I. Kh. Sabitov, Isometric transformations of a surface that generate its conformal mappings onto itself, volume 189, Mat. sb., 1998, No. 1, pp. 119-132. doi: https://doi.org/10.4213/sm297

[11] M. Stavrić, M. Manahl, A. Wiltsche, Discretization of double curved surface, Challenging Glass 4 \& COST Action TU0905 Final Conference - Louter, Bos \& Belis (Eds), Taylor \& Francis Group, London, 2014, pp. 133-140. doi:10.1201/b16499-23 